
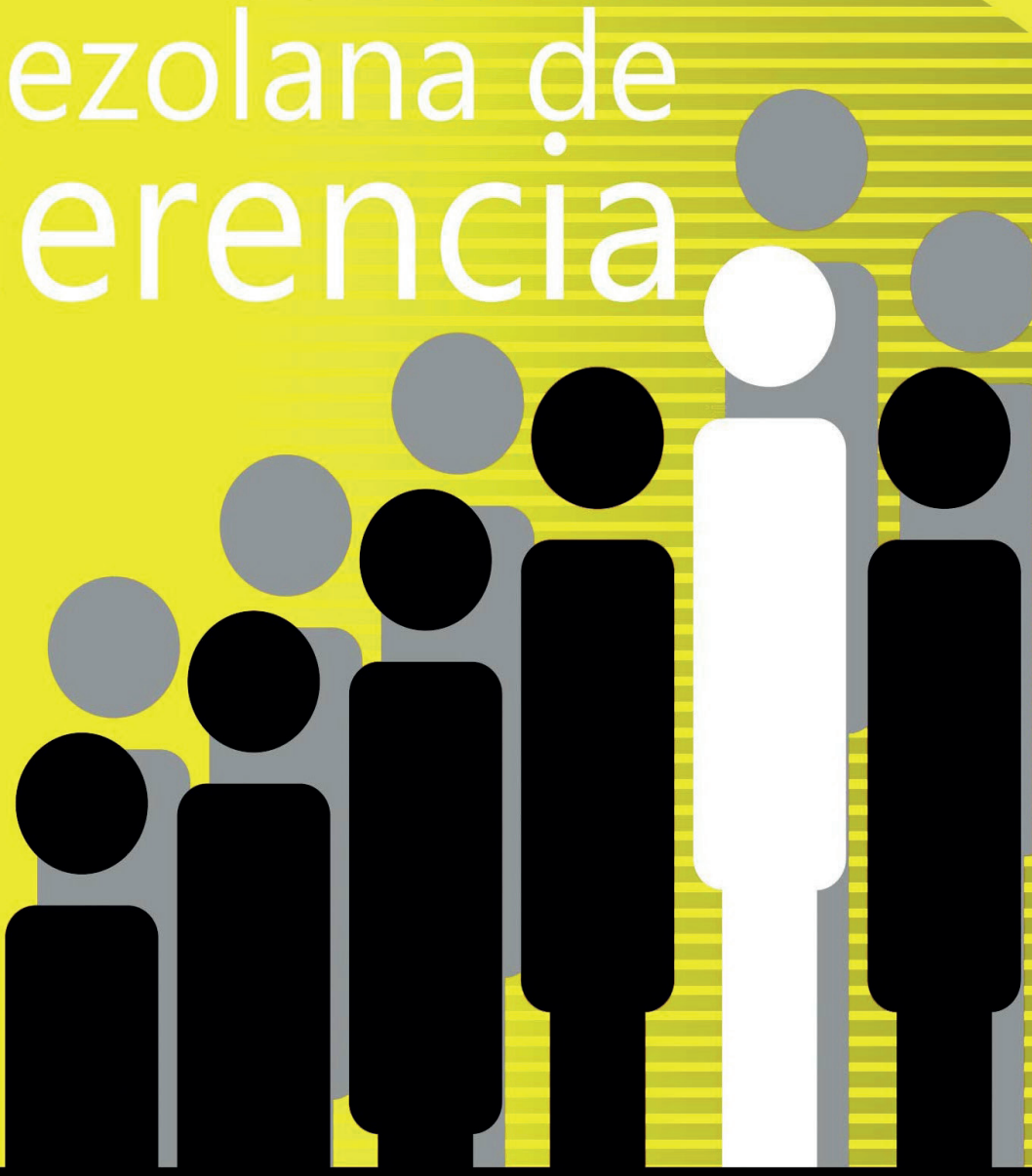


\title{
Escala de perfil emprendedor en estudiantes universitarios peruanos
}

\author{
Castillo Saavedra, Ericson Felix \\ Reyes Alfaro, Cecilia Elizabeth \\ Ayala Jara, Carmen Isolina ${ }^{* *}$ \\ Arroyo Rosales, Elizabeth Madeleine ${ }^{\star \star \star *}$
}

\section{Resumen}

Las universidades deben tener un instrumento que permita medir el perfil emprendedor del futuro profesional, de manera que pueda acceder rápidamente al entorno laboral. El estudio evaluó la escala de perfil emprendedor en universitarios peruanos mediante el análisis psicométrico de validez y confiabilidad. Se adaptó un cuestionario validado previamente en otro contexto y se aplicó a 354 estudiantes de una universidad pública de Trujillo (Perú) con edades de 19 a 23 años. Se determinó la validez mediante la evaluación del contenido y constructo; mientras que, para la confiabilidad, se utilizó el Alfa de Cronbach. Se encontró un V Aiken de 0,94; la prueba de Kaiser-Meyer-Olkin y la prueba de esfericidad de Bartlett arrojó un valor mayor a 0,5; comunalidades con valores mínimo y máximo de 0,586 y 0,881; con la identificación de 9 dimensiones, analizados por descomposición de la varianza total $(73,942 \%)$ y componentes rotados; en el análisis de correlación se encontró diferencia significativa en la dimensión - test, ítem - dimensión e ítem - test. En la confiabilidad se encontró un valor de Alfa de Cronbach de 0,833 . Se concluye que, la escala de perfil emprendedor es válido y confiable para el contexto peruano.

Palabras clave: Perfil emprendedor; escala; psicometría.

\section{Recibido: 15.11.20 Aceptado: 20.03.21}

* Doctor en Ciencias Biomédicas. Químico Farmacéutico. Docente en la Universidad Nacional de Trujillo, Perú. E-mail: ecastillos@unitru.edu.pe ORCID: https://orcid.org/0000-0002-9279-7189

** Doctora en Salud Pública. Licenciada en Enfermería. Docente en la Universidad César Vallejo, Perú. E-mail: creyesal@ucv.edu.pe ORCID: https://orcid.org/0000-0002-3528-546X

*** Doctora en Farmacia y Bioquímica. Químico Farmacéutica. Docente Principal en la Universidad Nacional de Trujillo, Perú. E-mail: cayala@unitru.edu.pe ORCID: https://orcid.org/0000-0002-4926-6497

**** Doctora en Gestión y Ciencias de la Educación. Licenciada en Educación. Docente en la Universidad César Vallejo, Perú. ORCID: https://orcid.org/0000-0002-0586-8429 Email; marlogi68@yahoo.es 


\title{
Entrepreneur profile scale in Peruvian university students
}

\begin{abstract}
Universities must have an instrument that allows to measure the entrepreneurial profile of the future professional that allows quick access to the work environment. The study evaluated the entrepreneurial profile scale in Peruvian university students through the psychometric analysis of validity and reliability. A previously validated questionnaire in another context was adapted and applied to 354 students from a public university in Trujillo (Peru) aged 19 to 23 years. Validity was determined by evaluating the content and construct; while, for reliability, Cronbach's Alpha was used. A V Aiken of 0.94 was found; the Kaiser-Meyer-Olkin test and the Bartlett sphericity test yielded a value greater than 0.5 ; communalities with minimum and maximum values of 0.586 and 0.881 ; with the identification of 9 dimensions, analyzed by decomposition of the total variance $(73.942 \%)$ and rotated components; In the correlation analysis, a significant difference was found in the dimension - test, item - dimension and item - test. In reliability, a Cronbach's Alpha value of 0.833 was found. It is concluded that the entrepreneur profile scale is valid and reliable for the Peruvian context.
\end{abstract}

Keywords: Entrepreneurial profile; scale; psychometry.

\section{Introducción}

La globalización, los desarrollos tecnológicos y el acelerado ritmo en la que se está viviendo, tienen un efecto irreversible sobre el aspecto social, económico, político y cultural de la humanidad. En ese sentido, la manera de afrontar estos cambios es mediante la creación de nuevos modelos, que solo pueden surgir a partir del emprendimiento, que ha sido considerado como el factor principal y desencadenante del desarrollo económico, permitiendo ascender socialmente a diversos sectores de la población (Roca, 2020).

Al respecto, estos cambios influyen significativamente en la educación universitaria, atribuyéndole de ese modo dos funciones; la primera en donde es la responsable de la evaluación, toma de decisiones y análisis respecto a las ventajas e inconvenientes que se podrían generar, $y$, la segunda, que es la de ajustarse de acuerdo a los nuevos requerimientos (Avendaño y Guacaneme, 2016; Capella, et al, 2016). En consecuencia, se han suscitado también cambios en las relaciones que se dan entre las empresas, el estado y las universidades (Rengifo, 2015).

La Organización Internacional del Trabajo (OIT) refiere en el 2018, que 3300 millones de personas económicamente activas no gozaban de seguridad económica, bienestar material e igualdad de oportunidades, y que paradójicamente, la disminución en la tasa de desempleo no se reflejaba en una mejora de la calidad del trabajo (OIT, 2019). 
Actualmente, ante esta situación de crisis económica y desempleo manifestada en diversos países, se ha suscitado una fascinación por las personas con rasgos de personalidad emprendedora, dado que tales rasgos de emprendimiento generan impacto positivo sobre la institución a la que pertenecen. En este sentido, Ward, et al (2019) expresa que todo profesional egresado de las aulas universitarias, debe poseer habilidades de emprendimiento, que promuevan el desarrollo económico y el posicionamiento en cargos directivos que permitan tomar decisiones asertivas en beneficio de la institución y del grupo humano que dirige.

De esta forma, se considera como factor de éxito para un futuro profesional poseer habilidades para el emprendimiento, y para ello es necesario conocer las características psicológicas de las personas emprendedoras, considerado como esencial para la generación de fuentes de trabajo, $y$, por tanto, del desarrollo económico mundial (Obschonka, et al, 2018; Razmus y Laguna, 2018; Sánchez, 2010; Suárez y Pedrosa, 2016).

En este sentido, los estudiantes universitarios representarían la parte productiva de un país a corto o mediano plazo, y mediante los ingresos económicos que generen cuando lleguen a ser profesionales, se sostendrá el desarrollo en infraestructura, ciencia y tecnología, condiciones sanitarias, entre otras situaciones.

Por consiguiente, se hace necesario, que en las universidades se realice un diagnóstico del perfil emprendedor que presentan los estudiantes, y de esta manera, asegurar que esta población que será económicamente activa se inserte rápidamente a puestos laborales como trabajador dependiente, o en caso de no acceder, genere emprendimientos independientes relacionados a su área profesional. Para ello, se requiere validar psicométricamente un instrumento que permita medir el perfil de emprendimiento, enfocado en universitarios de ciclos superiores, y generar alternativas de solución y/o reforzamiento en aquellos estudiantes que no presenten el perfil requerido.

El estudio se basó en una metodología de tipo instrumental y estuvo constituido por 354 estudiantes universitarios de una universidad pública de Perú, con edades de 19 a 23 años, seleccionados por muestreo no probabilístico por conveniencia. El programa SPSS versión 25 se utilizó para realizar el análisis multivariante de la validez de constructo, mediante el cálculo de los componentes principales y de tipo factorial. Dentro de las pruebas destinadas para este propósito se encuentran Kaiser-MeyerOlkin (KMO), esfericidad de Bartlett, extracción de comunalidades, gráfico de sedimentación y componentes rotados. De la misma forma, en la validez de contenido se utilizó la prueba juicio de expertos mediante el cálculo de $\mathrm{V}$ de Aiken, mientras que, en la validez de criterio, las técnicas correlacionales, a través del cálculo del coeficiente de Spearman. Por otra parte, la confiabilidad se determinó mediante el coeficiente Alfa de Cronbach.

\section{Emprendimiento, personalidad emprendedora: consideraciones teóricas}

El emprendimiento es la capacidad que consiste en crear y dar inicio a un nuevo propósito, involucrando a todas las 
actividades administrativas y logísticas para lograr y mantener el éxito. Asimismo, se resalta la importancia de que todo emprendimiento involucra aspectos de tipo gerencial orientado hacia la generación de un negocio, que tiene como finalidad ofertar bienes, servicios y nuevas oportunidades de trabajo. Estos aspectos de emprendimiento deben motivarse desde la universidad, porque existen algunas carreras profesionales que pueden incursionar en el ámbito empresarial, pero para ello, se debe tener conocimientos sobre criterios administrativos y/o gerenciales.

Por otro lado, las funciones de los emprendedores no son estables, es decir, cambian constantemente de acuerdo a las demandas del negocio, de tal forma que pueden ir desde formar la idea de negocio, elaborar planes estratégicos, y, finalmente su implementación. Por tanto, el emprendimiento siempre trae consigo cambios en la forma de pensamiento.

La personalidad emprendedora es un rasgo o característica intrínseca o adquirida que presenta un determinado sujeto para lograr un determinado fin en un tiempo previsto. Al respecto, para la formación de emprendedores es necesario estudiar la personalidad emprendedora, así como los factores que promueven o lo obstaculicen, dentro de ellos se pueden citar a las barreras internas, ambiente externo, entre otros. De otra manera, es necesario el estudio holístico de la personalidad emprendedora, teniendo en consideración la influencia de los factores socioculturales e individuales que la promueven. Al respecto, Przepiorka (2017) y Rojas, et al, (2019) describen que el emprendimiento ha causado diversas modificaciones a la estructura curricular de las universidades, sin embargo, es aún materia que se encuentra en desarrollo. Por su parte, Shu et al, (2019) y Tarapuez, et al, (2015) refieren que se han hallado obstáculos para implementar un modelo de educación basada en el emprendimiento que sincronice los conocimientos adquiridos con las necesidades reales del mercado laboral.

De este modo, se presentan características que definen una personalidad emprendedora, entre ellas se mencionan a la motivación de logro, autonomía, innovación, locus de control interno, autoeficacia, optimismo, tolerancia al estrés y toma de riesgos (Malavé y Piñango, 2012; Zapata et al, 2019; Zapata y Hernández, 2017). Las personas que cuentan con estos rasgos son precursores del crecimiento económico y éxito empresarial, por ende, es de suma importancia poder contar con recursos para identificar a los emprendedores, los cuales están dotados de una personalidad característica que desencadena su comportamiento emprendedor con el cual se propone, se ejecuta y mantiene cualquier proyecto a corto, mediano y largo plazo (Fellnhofer, 2017; Martínez et al, 2018; Molino et al, 2018; Sánchez, 2010; Sánchez, et al, 2017).

\section{Evaluación psicométrica del test de perfil emprendedor del estudiante universitario}

La evaluación psicométrica representa la forma de categorizar y evaluar un instrumento de medición de acuerdo al contexto problemático donde se desarrolla el estudio, y se fundamenta en análisis matemáticos computarizados, que asignan valores cuantitativos a objetos (Cuesta, et al, 2018; Mahmoudian et al, 2018; Rosales 
et al, 2017).

La validez y la confiabilidad representan los parámetros implicados en una evaluación psicométrica. La validez se refiere a la forma como el instrumento está midiendo el atributo que pretende medir, y a su vez se subdivide en contenido, constructo y criterio. En la validez de contenido, se evalúa por parte de un equipo denominado juicio de expertos, si las preguntas que conforman el instrumento, son importantes para la utilización que se le va a dar, es decir, si está relacionado a la temática a abordar (Capella et al, 2016; Cuesta et al, 2018). Por su parte, la validez de constructo, sistematiza y mide los comportamientos que registra el instrumento, de tal forma, que se consideren válidos del constructo al cual refieren (Berrios et al, 2019; Díaz, 2018). Finalmente, en la validez de criterio se establecería una relación entre los puntajes obtenidos por el instrumento en comparación con otro considerado como referencia (Flores et al, 2017; Galicia et al, 2016).

La confiabilidad se define como el grado de precisión con el que una prueba mide lo que realmente desea medir, en función de la concordancia de las mediciones realizadas y de las condiciones normales de aplicación. Para ello, es necesario la evaluación de la consistencia interna, que permite medir la correlación existente entre las diferentes preguntas que contiene el instrumento (Leal et al, 2016; Pucker et al, 2018; Rosales et al, 2017; Sánchez, 2010).

En una búsqueda exhaustiva en base de datos de alto impacto se evidenció la ausencia de un instrumento que permita medir el perfil de personalidad emprendedora, teniendo en consideración las características de los estudiantes universitarios peruanos (innovadores, perseverantes, resilientes y visionarios), debiendo incidir en la importancia de contar con un cuestionario idóneo para una determinada población, garantizando su validez de contenido, criterio y constructo, así como una alta confiabilidad.

Diversos estudios como Capella et al, (2016), Cuesta et al, (2018), Rojas et al, (2019), Sánchez et al, (2017), Tarapuez et al, (2015) y Ward et al, (2019) han propuesto instrumentos para medir el emprendimiento, con distintos enfoques como el social, así como también, la medición de las características de la personalidad emprendedora. En términos generales, los instrumentos diseñados y validados en diferentes contextos fueron orientados a la población estudiantil universitaria.

De este modo, al evaluar las características socioeconómicas y culturales del contexto peruano seleccionaron el Test Adaptativo Informatizado (TAI) tomado de Pedrosa (2015), constituido por 107 preguntas agrupadas en 9 rasgos específicos (autoeficacia, motivación de logro, optimismo, innovación, autonomía, toma de riesgos, locus de control interno y tolerancia al estrés). El TAl permite tener un instrumento de medición adecuado que reduce la incertidumbre sobre el nivel de riesgo de cada persona evaluada, maximizando la precisión de las estimaciones (Pedrosa, 2015; Suárez y Pedrosa, 2016).

Por tanto, en base a las características de la personalidad emprendedora se consideró necesarias utilizarlas como dimensiones o componentes en una evaluación psicométrica que tiene como objeto de estudio el emprendimiento.

La investigación se realizó en la ciudad de Trujillo (Perú) y estuvo 
conformada por 354 estudiantes de una universidad pública, seleccionados por muestreo no probabilístico por conveniencia, de acuerdo al mismo ciclo de estudio y de la facultad donde se encontraban registrados, de tal manera, que se pueda tener una mayor fiabilidad de los resultados obtenidos.

Los estudiantes de universidades públicas provienen de diferentes estratos socioeconómicos, donde la necesidad de dinero es recurrente, sin embargo, su motivación de progresar es mayor que sus carencias, por tal motivo, es necesario que se puedan implementar asignaturas de emprendimiento, porque se debe considerar, que no todos los egresados logran acceder a un puesto laboral, y optarían por un trabajo independiente. El asegurar una política de emprendimiento a nivel universitario posibilita que mayor cantidad de profesionales se inserten al campo laboral, generando el crecimiento económico de una nación.

La tabla 1 mide la validez de contenido mediante el método a juicio de expertos, que consiste en un examen sistemático de cada de uno de los ítems-preguntas, para determinar si miden las dimensiones que se espera medir, la pertinencia del contenido, la claridad de los enunciados de cada uno de ellos, y el nivel de concordancia entre los evaluadores. El método matemático utilizado en el estudio fue el $\checkmark$ Aiken politómica, prueba ampliamente utilizada en el análisis psicométrico que cumplió con los criterios de elaboración de acuerdo a la opción de respuesta. El valor de 0,94 reflejaría que los 5 jueces estuvieron de acuerdo con el contenido de los ítems, y según como indica la literatura, valores mayores a 0,90 son indicativos de una adecuada validación de contenido (Galicia, et al, 2017).

\section{Tabla 1}

\section{Validez de contenido del test de perfil emprendedor según juicio de experto $(n=5)$}

\begin{tabular}{|c|c|c|c|c|c|c|c|c|}
\hline \multirow{2}{*}{\multicolumn{2}{|c|}{ Item }} & \multicolumn{5}{|c|}{ Calificaciones } & \multirow{2}{*}{ Suma } & \multirow{2}{*}{$\mathbf{V}$} \\
\hline & & 1 & 2 & 3 & 4 & 5 & & \\
\hline $\begin{array}{l}1 . \\
\text { edor }\end{array}$ & Aprovecho los recursos que tengo a mi alred- & 0 & 1 & 1 & 1 & 1 & 4 & 0,80 \\
\hline 2. & Creo que puedo superar cualquier reto & 1 & 1 & 1 & 1 & 1 & 5 & 1,00 \\
\hline $\begin{array}{l}3 . \\
\text { capacidad }\end{array}$ & Me gustan los retos que ponen a prueba mi & 1 & 0 & 1 & 1 & 1 & 4 & 0,80 \\
\hline 4. & Resuelvo las tareas de forma eficaz & 1 & 1 & 1 & 1 & 0 & 4 & 0,80 \\
\hline 5. & Me gusta organizar mi tiempo de trabajo & 1 & 1 & 1 & 1 & 1 & 5 & 1,00 \\
\hline $\begin{array}{l}6 . \\
\text { rendiradecu }\end{array}$ & $\begin{array}{l}\text { Necesito que alguien controle mi trabajo para } \\
\text { uadamente }\end{array}$ & 1 & 1 & 1 & 1 & 1 & 5 & 1,00 \\
\hline 7. & Apoyo las ideas nuevas & 1 & 1 & 1 & 1 & 1 & 5 & 1,00 \\
\hline 8. & Me gusta aprender nuevas formas de trabajar & 1 & 1 & 1 & 1 & 1 & 5 & 1,00 \\
\hline 9. & Me gusta mejorar las cosas & 1 & 1 & 1 & 1 & 1 & 5 & 1,00 \\
\hline
\end{tabular}




\section{Cont... Tabla 1}

\begin{tabular}{|c|c|c|c|c|c|c|c|c|}
\hline $\begin{array}{l}10 . \\
\text { tase cosas }\end{array}$ & $\begin{array}{l}\text { Me gustaría trabajar en una empresa que apor- } \\
\text { nuevas al mercado }\end{array}$ & 1 & 1 & 1 & 1 & 1 & 5 & 1,00 \\
\hline $\begin{array}{l}11 . \\
\text { compañero }\end{array}$ & $\begin{array}{l}\text { Planteo soluciones diferentes a las de mis } \\
\text { S }\end{array}$ & 1 & 1 & 1 & 1 & 1 & 5 & 1,00 \\
\hline 12. & Veo diferentes soluciones a los problemas & 1 & 1 & 1 & 1 & 1 & 5 & 1,00 \\
\hline $\begin{array}{l}13 . \\
\text { ciativa }\end{array}$ & Prefiero que sean otros quienes toman la ini- & 1 & 1 & 1 & 1 & 0 & 4 & 0,80 \\
\hline $\begin{array}{l}14 . \\
\text { estudiado }\end{array}$ & La nota de un examen depende de lo que hayas & 1 & 1 & 1 & 1 & 1 & 5 & 1,00 \\
\hline 15. & El éxito depende de mi esfuerzo & 1 & 1 & 1 & 1 & 1 & 5 & 1,00 \\
\hline 16. & Mis éxitos son consecuencia de mi esfuerzo & 1 & 1 & 1 & 1 & 1 & 5 & 1,00 \\
\hline 17. & Mi futuro depende de lo que yo haga & 1 & 1 & 1 & 1 & 1 & 5 & 1,00 \\
\hline 18. & Para alcanzar una meta es necesario esforzarse & 1 & 1 & 1 & 0 & 1 & 4 & 0,80 \\
\hline 19. & Aspiro a ser el mejor en mi trabajo & 1 & 1 & 1 & 1 & 1 & 5 & 1,00 \\
\hline $\begin{array}{l}20 . \\
\text { un esfuerzo }\end{array}$ & $\begin{array}{l}\text { Cuando tengo miedo de cometer errores hago } \\
\text { o extra }\end{array}$ & 1 & 1 & 1 & 1 & 1 & 5 & 1,00 \\
\hline 21. & Pienso que todo saldrá mal & 1 & 1 & 1 & 1 & 1 & 5 & 1,00 \\
\hline 22. & Tengo confianza en superar los problemas & 1 & 1 & 1 & 1 & 1 & 5 & 1,00 \\
\hline 23. & Hay pocas situaciones que me generen estrés & 1 & 1 & 1 & 1 & 1 & 5 & 1,00 \\
\hline 24. & Me agobia el futuro & 1 & 0 & 1 & 1 & 1 & 4 & 0,80 \\
\hline 25. & Me estreso con facilidad & 1 & 1 & 1 & 0 & 1 & 4 & 0,80 \\
\hline 26. & Me bloqueo cuando tengo mucho trabajo & 1 & 1 & 1 & 0 & 1 & 4 & 0,80 \\
\hline $\begin{array}{l}27 . \\
\text { por pequeñ }\end{array}$ & $\begin{array}{l}\text { Evito las situaciones que implican algún riesgo } \\
\text { ño que sea }\end{array}$ & 1 & 1 & 1 & 1 & 1 & 5 & 1,00 \\
\hline 28. & No asumir riesgos es de cobardes & 1 & 1 & 1 & 1 & 1 & 5 & 1,00 \\
\hline \multicolumn{2}{|c|}{$\begin{array}{l}29 . \quad \text { Soy capaz de renunciar a cosas que ya tengo } \\
\text { por alcanzar un objetivo mejor }\end{array}$} & 1 & 1 & 1 & 1 & 1 & 5 & 1,00 \\
\hline & V Aiken & & & & & & & 0,94 \\
\hline
\end{tabular}

Fuente: Elaboración propia

De esta manera, un juicio de expertos constituido por un grupo selecto de profesionales en el rubro de empresarial permite evaluar las características requeridas por un estudiante universitario peruano para crear en un primer momento pequeñas y medianas empresas a corto y mediano plazo, y posteriormente, a medida que se incrementen las utilidades poder expandirse a otras latitudes (Martínez et al, 2018; Molino et al, 2018). 
Al mismo tiempo, se debe incentivar en las aulas universitarias, aspectos de invención, que no necesariamente pueden ser aspectos complejos, sino útiles y necesarios para la sociedad, para que posteriormente puedan registrarse como patente ante el organismo oficial de cada país.

En la tabla 2 se presenta el índice de KMO, que sirvió para evaluar las magnitudes de los coeficientes de correlación general con respecto a las magnitudes de los coeficientes de correlación parcial. Los valores de KMO entre 0,5 y 1 indican que es apropiado aplicar el análisis factorial a la matriz de datos. En el presente estudio se obtuvo un KMO de 0,534 lo que indica que la muestra seleccionada es apropiada y se puede continuar con la aplicación del análisis factorial.

\section{Tabla 2 \\ Validez de constructo del test de perfil emprendedor según la prueba de Kaiser-Meyer-Olkin y la prueba de esfericidad de Bartlett}

\begin{tabular}{lll}
\hline \multicolumn{2}{l}{ Prueba de KMO y Bartlett } & \\
\hline Medida Kaiser-Meyer-Olkin de adecuación de muestreo & 0,534 \\
& Aprox. Chi-cuadrado & 6850,349 \\
Prueba de esfericidad de Bartlett & GI & 406 \\
& Sig. & 0,000 \\
\hline
\end{tabular}

Fuente: Elaboración propia

Por otro lado, valores bajos con el índice $\mathrm{KMO}$, indicaría que las correlaciones entre pares de variables no pueden ser explicadas por las otras variables $y$, por lo tanto, no es factible llevar a cabo el análisis factorial, y que el índice KMO se alejará de cero (Flores et al, 2017).

De la misma forma, el test de esfericidad de Bartlett consistió en una estimación de ji-cuadrado a partir de una transformación del determinante de la matriz de correlaciones. Si las dimensiones o componentes no están intercorrelacionadas, entonces el test de esfericidad de Bartlett debe presentar un valor de significancia superior a 0,05 (Leal et al, 2016).

El test de personalidad emprendedora presentó una significancia muy inferior al límite 0,05 $(p=0,000)$, que indicaría que la matriz de datos de la validez de constructo es válida para continuar con el proceso de análisis factorial, es congruente con las subescalas establecidas, y apto para la medición del emprendimiento.

De la misma forma, en la tabla 3 se observa un análisis que se deriva de la evaluación de la tabla de comunalidades, que expone la proporción de varianza de cada dimensión o ítem explicada por el conjunto de factores comunes, donde a mayor sea la cantidad de comunalidades cercanas a 1 , mejor será la calidad de ajuste del modelo. El estudio presentó un conjunto de valores cercanos entre sí, con 0,586 y 0,881 para los valores mínimo y máximo respectivamente. 
Castillo Saavedra, Ericson Felix; Reyes Alfaro, Cecilia Elizabeth; Ayala Jara, Carmen Isolina; Arroyo Rosales, Elizabeth Madeleine Escala de perfil emprendedor en estudiantes universitarios peruanos

\section{Tabla 3}

Validez de constructo del test de perfil emprendedor según comunalidades

\begin{tabular}{|c|c|c|}
\hline Item & Inicial & Extracción \\
\hline 1 & 1,000 & 0,602 \\
\hline 2 & 1,000 & 0,720 \\
\hline 3 & 1,000 & 0,660 \\
\hline 4 & 1,000 & 0,687 \\
\hline 5 & 1,000 & 0,689 \\
\hline 6 & 1,000 & 0,637 \\
\hline 7 & 1,000 & 0,837 \\
\hline 8 & 1,000 & 0,636 \\
\hline 9 & 1,000 & 0,586 \\
\hline 10 & 1,000 & 0,676 \\
\hline 11 & 1,000 & 0,770 \\
\hline 12 & 1,000 & 0,822 \\
\hline 13 & 1,000 & 0,710 \\
\hline 14 & 1,000 & 0,699 \\
\hline 15 & 1,000 & 0,821 \\
\hline 16 & 1,000 & 0,865 \\
\hline 17 & 1,000 & 0,877 \\
\hline 18 & 1,000 & 0,881 \\
\hline 19 & 1,000 & 0,734 \\
\hline 20 & 1,000 & 0,717 \\
\hline 21 & 1,000 & 0,687 \\
\hline 22 & 1,000 & 0,721 \\
\hline 23 & 1,000 & 0,712 \\
\hline 24 & 1,000 & 0,818 \\
\hline 25 & 1,000 & 0,851 \\
\hline 26 & 1,000 & 0,854 \\
\hline 27 & 1,000 & 0,683 \\
\hline 28 & 1,000 & 0,775 \\
\hline 29 & 1,000 & 0,713 \\
\hline
\end{tabular}

Fuente: Elaboración propia 
Por consiguiente, Pucker (2018) establece que esta forma de extracción permite estimar la cercanía de los datos en función de las respuestas emitidas por los participantes y de esta manera predecir los componentes principales que serán necesarios para agrupar los 29 ítems que presenta el presente instrumento.

Por tanto, el análisis de comunalidades garantiza los resultados encontrados mediante la prueba de Kaiser-Meyer-Olkin y esfericidad de Bartlett.

La tabla 4 presenta la descomposición de la varianza por parte de los distintos factores, y se evidencia que 8 componentes explican el 69,018\% de la varianza total, mientras que el resto de los factores solamente aportan con el $30,982 \%$, por lo que debiesen ser desechados.

\section{Tabla 4}

Validez de constructo del test de perfil de personalidad emprendedora en estudiantes de una universidad pública según la varianza total explicada

\begin{tabular}{|c|c|c|c|c|c|c|c|c|c|}
\hline \multirow{2}{*}{$\mathrm{N}^{\circ}$} & \multicolumn{3}{|c|}{ Autovalores iniciales } & \multicolumn{3}{|c|}{$\begin{array}{l}\text { Sumas de extracción de cargas } \\
\text { al cuadrado }\end{array}$} & \multicolumn{3}{|c|}{$\begin{array}{l}\text { Sumas de rotación de cargas al } \\
\text { cuadrado }\end{array}$} \\
\hline & Total & $\begin{array}{c}\% \text { de } \\
\text { varianza }\end{array}$ & $\begin{array}{c}\% \\
\text { acumulado }\end{array}$ & Total & $\begin{array}{c}\% \text { de } \\
\text { varianza }\end{array}$ & $\begin{array}{c}\% \\
\text { acumulado }\end{array}$ & Total & $\begin{array}{c}\% \text { de } \\
\text { varianza }\end{array}$ & $\begin{array}{c}\% \\
\text { acumulado }\end{array}$ \\
\hline 1 & 4,871 & 16,797 & 16,797 & 4,871 & 16,797 & 16,797 & 3,499 & 12,066 & 12,066 \\
\hline 2 & 3,483 & 12,010 & 28,808 & 3,483 & 12,010 & 28,808 & 3,361 & 11,590 & 23,656 \\
\hline 3 & 3,004 & 10,358 & 39,166 & 3,004 & 10,358 & 39,166 & 2,893 & 9,975 & 33,630 \\
\hline 4 & 2,311 & 7,968 & 47,133 & 2,311 & 7,968 & 47,133 & 2,350 & 8,103 & 41,733 \\
\hline 5 & 2,040 & 7,034 & 54,167 & 2,040 & 7,034 & 54,167 & 2,092 & 7,214 & 48,948 \\
\hline 6 & 1,640 & 5,655 & 59,822 & 1,640 & 5,655 & 59,822 & 2,014 & 6,944 & 55,892 \\
\hline 7 & 1,550 & 5,344 & 65,166 & 1,550 & 5,344 & 65,166 & 1,966 & 6,781 & 62,672 \\
\hline 8 & 1,338 & 4,613 & 69,780 & 1,338 & 4,613 & 69,780 & 1,840 & 6,345 & 69,018 \\
\hline 9 & ,996 & 4,163 & 73,942 & & & & & & \\
\hline 10 & ,913 & 3,388 & 77,331 & & & & & & \\
\hline 11 & ,852 & 2,939 & 80,270 & & & & & & \\
\hline 12 & 787 & 2,714 & 82,984 & & & & & & \\
\hline 13 & ,696 & 2,401 & 85,386 & & & & & & \\
\hline 14 & 654 & 2,254 & 87,640 & & & & & & \\
\hline 15 & ,540 & 1,863 & 89,502 & & & & & & \\
\hline 16 & ,504 & 1,738 & 91,240 & & & & & & \\
\hline
\end{tabular}




\section{Cont... Tabla 4}

\begin{tabular}{llll}
\hline 17 &, 444 & 1,532 & 92,772 \\
18 &, 381 & 1,313 & 94,084 \\
19 &, 322 & 1,111 & 95,196 \\
20 &, 264 &, 910 & 96,106 \\
21 &, 224 &, 772 & 96,878 \\
22 &, 217 &, 749 & 97,627 \\
23 &, 162 &, 559 & 98,186 \\
24 &, 158 &, 546 & 98,732 \\
25 &, 129 &, 445 & 99,177 \\
26 &, 083 &, 287 & 99,463 \\
27 &, 063 &, 218 & 99,681 \\
28 &, 052 &, 181 & 99,862 \\
29 &, 040 &, 138 & 100,000 \\
\hline
\end{tabular}

Fuente: Elaboración propia

En un análisis factorial exploratorio es necesario obtener la cantidad de dimensiones en las cuales se agruparán los 29 ítems, y de esta manera el instrumento sirva de base para su aplicación en diferentes contextos. Para Portuguez et al, (2018), la estimación de la validez de constructo es un requisito indispensable para conocer si el instrumento que ha sido elaborado por primera vez es congruente entre sus dimensiones y sus respectivos ítems.

Asimismo, Rosales (2017) indica que la selección de los factores utilizando el método de los componentes principales consideró los valores propios (autovalores) mayores que 1 (valores propios >1). En este sentido, la tabla 4 indicaría que se deben extraer 8 componentes principales que son los que cumplen con el requisito señalado.
Sánchez (2010) evaluó un cuestionario de emprendimiento, donde identificó 8 dimensiones (autoeficacia para crear productos, autoeficacia para desarrollar un entorno laboral en el que los demás intenten hacer cosas nuevas, locus de control externo, proactividad, creatividad, suerte, locus de control interno y riesgo), similares a las del presente estudio, corroborando con ello, la fiabilidad del instrumento para el contexto peruano.

La tabla 5 muestra la matriz de componentes rotados, que puede determinar con mayor facilidad la forma de incluir cada variable en cada factor (componente). Para ello hay que observar para cada uno de los componentes, cuáles son las variables que presentan valores más altos. 
pp. $840-858$

Revista Venezolana de Gerencia, Año 26 No. 94, 2021

Tabla 5

Validez de constructo del test de perfil emprendedor según matriz de componente rotado

\begin{tabular}{|c|c|c|c|c|c|c|c|c|}
\hline \multirow{2}{*}{ Item } & \multicolumn{8}{|c|}{ Componente rotado } \\
\hline & 1 & 2 & 3 & 4 & 5 & 6 & 7 & 8 \\
\hline 1 & 0,918 & & & & & & & \\
\hline 2 & 0,877 & & & & & & & \\
\hline 3 & 0,873 & & & & & & & \\
\hline 4 & 0,749 & & & & & & & \\
\hline 5 & & 0,891 & & & & & & \\
\hline 6 & & 0,846 & & & & & & \\
\hline 7 & & & 0,803 & & & & & \\
\hline 8 & & & 0,786 & & & & & \\
\hline 9 & & & 0,747 & & & & & \\
\hline 10 & & & 0,803 & & & & & \\
\hline 11 & & & 0,747 & & & & & \\
\hline 12 & & & 0,671 & & & & & \\
\hline 13 & & & 0,490 & & & & & \\
\hline 14 & & & & 0,826 & & & & \\
\hline 15 & & & & 0,770 & & & & \\
\hline 16 & & & & 0,769 & & & & \\
\hline 17 & & & & 0,606 & & & & \\
\hline 18 & & & & 0,559 & & & & \\
\hline 19 & & & & & 0,775 & & & \\
\hline 20 & & & & & 0,689 & & & \\
\hline 21 & & & & & & 0,791 & & \\
\hline 22 & & & & & & 0,770 & & \\
\hline 23 & & & & & & & 0,788 & \\
\hline 24 & & & & & & & 0,760 & \\
\hline 25 & & & & & & & 0,522 & \\
\hline 26 & & & & & & & 0.507 & \\
\hline
\end{tabular}




\section{Cont... Tabla 5}

\begin{tabular}{ll}
\hline 27 & 0,782 \\
28 & 0,648 \\
29 & 0,562 \\
\hline
\end{tabular}

Fuente: Elaboración propia

De esta manera, según Capella et al, (2016) y Cuesta et al, (2018), los componentes rotados relacionan la variable a la conformación de un factor o dimensión; mientras más elevada sea la carga, mayor peso tiene en la interpretación del modelo. Las cargas factoriales menores a 0,3 están en el nivel mínimo y las mayores a 0,5, están en un nivel significativo.

Elestudio presenta conformaciones respecto a la similitud entre sus valores, de mayor (componente 1) a menor (componente 8), por lo que el instrumento tendrá 8 dimensiones en donde se agruparán los 29 ítems.

En la tabla 6 se presentan las técnicas correlacionales respecto a la relación existente entre las dimensiones y el test, se analizó mediante la correlación de Pearson y se encontró diferencia significativa para cada dimensión. Este resultado permite inferir que el instrumento presenta un elevado grado de congruencia en la elaboración de sus dimensiones.

Tabla 6

Validez de constructo del test de perfil emprendedor según correlación dimensión - test

\begin{tabular}{ll}
\hline \multicolumn{1}{c}{ Dimensión } & Correlación \\
\hline Motivación de logro & $0,377^{* *}$ \\
\hline Autonomía & $0,362^{* *}$ \\
\hline Innovación & $0,628^{* *}$ \\
\hline Locus de control interno & $0,384^{* *}$ \\
\hline Autoeficacia & $0,153^{* *}$ \\
\hline Optimismo & $0,586^{* *}$ \\
\hline Tolerancia al estrés & $0,562^{* *}$ \\
\hline Toma de riesgos & $0,536^{* *}$ \\
\hline **. La correlación es significativa en el nivel 0,01 (bilateral). \\
*. La correlación es significativa en el nivel 0,05 (bilateral). \\
Fuente: Elaboración propia
\end{tabular}


pp. $840-858$

Revista Venezolana de Gerencia, Año 26 No. 94, 2021

De esta manera, el grado significativo y muy significativo garantizaría la idoneidad de las dimensiones respecto a la variable que se está investigando, logrando una asociación armoniosa entre lo que se mide y sus ámbitos de acción.
La tabla 7 refleja los 8 componentes identificados con la validación del instrumento, siendo similares a lo reportado por Pedrosa (2015) al aplicar el TAl en jóvenes españoles.

Tabla 7

Validez de constructo del test de perfil emprendedor según correlación ítem - dimensión

\begin{tabular}{|c|c|c|}
\hline Dimensión & Ítem & Correlación \\
\hline \multirow{4}{*}{ Motivación de logro } & 1 & $0,519^{* *}$ \\
\hline & 2 & $0,692^{* *}$ \\
\hline & 3 & $0,620^{* *}$ \\
\hline & 4 & $0,581^{* *}$ \\
\hline \multirow{2}{*}{ Autonomía } & 5 & $0,645^{* *}$ \\
\hline & 6 & 0,732 \\
\hline \multirow{7}{*}{ Innovación } & 7 & $0,445^{* *}$ \\
\hline & 8 & $0,275^{* *}$ \\
\hline & 9 & $0,498^{* *}$ \\
\hline & 10 & $0,392^{* *}$ \\
\hline & 11 & $0,544^{* *}$ \\
\hline & 12 & $0,735^{* *}$ \\
\hline & 13 & $0,184^{* *}$ \\
\hline \multirow{5}{*}{ Locus de control interno } & 14 & $0,555^{* *}$ \\
\hline & 15 & $0,775^{* *}$ \\
\hline & 16 & $0,763^{* *}$ \\
\hline & 17 & 0,732 \\
\hline & 18 & $0,750^{* *}$ \\
\hline \multirow{2}{*}{ Autoeficacia } & 19 & $0,806^{* *}$ \\
\hline & 20 & $0,782^{* *}$ \\
\hline \multirow{2}{*}{ Optimismo } & 21 & $0,853^{* *}$ \\
\hline & 22 & $0,305^{* *}$ \\
\hline \multirow{4}{*}{ Tolerancia al estrés } & 23 & 0,050 \\
\hline & 24 & $0,815^{* *}$ \\
\hline & 25 & $0,820^{* *}$ \\
\hline & 26 & $0,810^{* *}$ \\
\hline
\end{tabular}


Castillo Saavedra, Ericson Felix; Reyes Alfaro, Cecilia Elizabeth; Ayala Jara, Carmen Isolina; Arroyo Rosales, Elizabeth Madeleine Escala de perfil emprendedor en estudiantes universitarios peruanos

\section{Cont... Tabla 7}

\begin{tabular}{lll}
\hline \multirow{3}{*}{ Toma de riesgos } & 27 & $0,529^{* *}$ \\
& 28 & $0,667^{* *}$ \\
& 29 & $0,379^{* *}$ \\
\hline
\end{tabular}

**. La correlación es significativa en el nivel 0,01 (bilateral).

*. La correlación es significativa en el nivel 0,05 (bilateral).

Fuente: Elaboración propia

De esta manera, se puede observar concordancia entre los diferentes contextos de aplicación. EI presente estudio permitió encontrar 29 preguntas que se distribuyeron en rasgos específicos de autoeficacia, motivación de logro, optimismo, innovación, autonomía, toma de riesgos, locus de control interno y tolerancia al estrés.

Las tablas 7 y 8 relacionaron los ítems planteados en el estudio con cada dimensión, y posteriormente con los valores totales; $y$ en todos los casos se evidenció diferencia altamente significativa.

\begin{tabular}{cc} 
Cont... Tabla 8 \\
\hline 15 & $0,377^{\star *}$ \\
16 & $0,371^{\star *}$ \\
17 & $0,253^{\star *}$ \\
18 & $0,283^{\star *}$ \\
19 & $0,149^{\star *}$ \\
20 & 0,93 \\
21 & $0,354^{\star *}$ \\
22 & $0,446^{\star *}$ \\
23 & 0,056 \\
24 & $0,403^{* *}$ \\
25 & $0,533^{\star *}$ \\
26 & $0,405^{\star *}$ \\
27 & $0,271^{* *}$ \\
28 & $0,383^{\star *}$ \\
29 & $0,186^{\star *}$ \\
\hline
\end{tabular}

**. La correlación es significativa en el nivel 0,01 (bilateral).

*. La correlación es significativa en el nivel 0,05 (bilateral).

Fuente: Elaboración propia 
De esta manera, Sánchez (2010) y Portuguez et al, (2018) encontraron resultados similares en sus técnicas correlacionales respecto al presente estudio, lo que indicaría que cuando se aplican instrumentos relacionados a emprendimiento, los estudiantes se sienten motivados en incursionar en ideas de negocio que permitan potenciar sus conocimientos y que a la vez generen ingresos económicos.

Finalmente, la tabla 9 presenta el resultado de Alfa de Cronbach, y refiere que cuanto más se aproxime a su valor máximo, 1, mayor es la fiabilidad de la escala, en determinados contextos, se considera que valores del alfa superiores a 0,7 o 0,8 (dependiendo de la fuente) son suficientes para garantizar la fiabilidad de la escala. En el estudio, se puede observar que el valor de alfa es de $0,872>0,70$, y se puede inferir que nuestro cuestionario es confiable $o$ aceptable.

\section{Tabla 9 \\ Confiabilidad del test de perfil de personalidad emprendedora en estudiantes de una universidad pública según Alfa de Cronbach}

\begin{tabular}{cc}
\hline Alfa de Cronbach & Elementos \\
\hline 0,833 & 29 \\
\hline
\end{tabular}

Fuente: Elaboración propia

Por su parte, Cuesta et al, (2018) al medir la fiabilidad de un cuestionario de emprendimiento encontró valores comprendidos entre 0,81 a 0,89 para las categorías, asegurando de esta forma, su aplicabilidad en contextos poblacionales.

De este modo, los cuestionarios relacionados a emprendimiento son necesarios sobre todo en ámbitos educativos, en donde se puede potenciar las habilidades, destrezas y capacidades de los futuros profesionales.

\section{Conclusiones}

El emprendimiento representa la forma como una persona en base a su esfuerzo logra sus objetivos a corto, mediano y largo plazo, es por ello que, todos los estudiantes, preferentemente en un nivel de educación superior deben integrar además de los conocimientos propios de su especialidad características de emprendedor, debido a que representan el sostenimiento económico de una nación.

El estudio enfoca un instrumento de perfil emprendedor validado mediante análisis psicométrico al contexto peruano, constituido por 29 preguntas y que presenta como dimensiones: motivación de logro, autonomía, innovación, locus de control interno, autoeficacia, optimismo y tolerancia al estrés; que permita a cada universidad diagnosticar el perfil emprendedor de un estudiante, y aplicar alternativas de solución en aquellos que no cumplan con el perfil requerido.

Dentro de las limitaciones se encuentran el número de estudiantes participantes en el estudio, por lo que se sugiere integrar a todas las facultades de las universidades públicas y privadas, para que, de esta forma, personas con educación de nivel superior, presenten un perfil emprendedor, que, a su vez, desarrollen habilidades y destrezas, con enfoque económico y de invención.

\section{Referencias bibliográficas}

Avendaño, W. y Guacaneme, R. 
Castillo Saavedra, Ericson Felix; Reyes Alfaro, Cecilia Elizabeth;

Ayala Jara, Carmen Isolina; Arroyo Rosales, Elizabeth Madeleine

Escala de perfil emprendedor en estudiantes universitarios peruanos

(2016). Educación y globalización: una visión crítica. Civilizar, 10(30), 191-206. https://doi. org/10.22518/16578953.543

Berrios, J., Frias, D., Pascual, M. y Muzatto, P. (2019). Escala de satisfacción con la vida: propiedades psicométricas de inmigrantes en España. Revista de Ciencias Sociales (Ve), 25(1), 130-142. https:// doi.org/10.31876/rcs.v25i1.27305

Capella, C., Gil, J., Martí, M. y Ruiz, P. (2016). Construcción de un cuestionario para medir el emprendimiento social en educación física. Pedagogía Social. Revista Interuniversitaria, 28, 169-188.

Cuesta, M., Suárez, J., Lozano, L., García, E. \& Muñiz, J. (2018). Assessment of eight entrepreneurial personality dimensions: Validity evidence of the BEPE battery. Frontiers in Psychology, 9, 2352.

Diaz, J. (2018). Políticas públicas en propiedad intelectual escrita. Una escala de medición para educación superior del Perú. Revista Venezolana de Gerencia, 23(81), 88105. https://doi.org/10.37960/revista. v23i81.23470

Fellnhofer, K. (2017). The power of passion in entrepreneurship education: entrepreneurial role models encourage passion?. Journal of entrepreneurship education, 20(1), 58-87.

Flores, O., Lajo, Y., Zevallos, A., Rondán, P., Lizaraso, F. \& Jorquiera, T. (2017). Psychometric analysis of a questionnaire to measure the educational environment in a sample of medical students in Peru. Revista Peruana de Medicina Experimental y Salud Pública, 34(2), 255-260. http://doi.org/10.17843/ rpmesp.2017.342.2642
Galicia, L., Balderrama, J. y Edel, R. (2017). Validez de contenido por juicio de expertos: propuesta de una herramienta virtual. Apertura (Guadalajara, Jal.), 9(2), 42-53.

Leal, C., Tirado, S., Van-der, C. y Rodríguez, J. (2016). Creación de la escala sobre habilidades de comunicación en profesionales de la salud, EHC-PS. Anales de Psicología, 32(1), 49-59. http://dx.doi. org/10.6018/analesps.31.3.184701

Mahmoudian, A., Torabi, R., Alipour, A., Shamsalinia, A. \& Ghaffari, F. (2018). The design and evaluation of psychometric properties for a questionnaire on elderly abuse by family caregivers among older adults on hemodialysis. Clinical interventions in aging, 13, 555-563. https://doi.org/10.2147/CIA.S149338

Malavé, J. y Piñango, R. (2012). Líderes, gerentes y emprendedores: un estudio empírico de actitudes y significados. Revista Venezolana de Gerencia, 17(60), 676-694. https://doi.org/10.37960/revista. v17i60.10938

Martínez, V., Cuesta, M., Lozano, L., Pedrosa, I. \& Muñiz, J. (2018). Multifactor implicit measures to assess enterprising personality dimensions. Psicothema, 30(4), 357-363. http://doi.org/10.7334/ psicothema2018.204

Molino, M., Dolce, V., Cortese, C. \& Ghislieri, C. (2018). Personality and social support as determinants of entrepreneurial intention. Gender differences in Italy. PloS one, 13(6), e0199924. https://doi.org/10.1371/ journal.pone.0199924

Obschonka, M., Moeller, J. \& Goethner, M. (2018). Entrepreneurial passion and personality: the case of academic entrepreneurship. Frontiers in Psychology, 9, 2697. https://doi. 


\section{org/10.3389/fpsyg.2018.02697}

Organización Internacional del Trabajo OIT (2019). Perspectivas sociales y del empleo en el mundo - Tendencias 2019. https://www.ilo.org/global/ about-the-ilo/newsroom/news/ WCMS 670577/lang--es/index.htm

Pedrosa, I. (2015). Evaluación de la personalidad emprendedora mediante un Test Adaptativo Informatizado. (Tesis doctoral). Universidad de Oviedo, Oviedo, España.

Pekkala, S., Kerr, W. \& Dalton, M. (2019). Risk attitudes and personality traits of entrepreneurs and venture team members. PNAS, 116(36). https:// doi.org/10.1073/pnas.1908375116

Portuguez, M., Valenzuela, J. y Navarro, C. (2018). Diseño y validación de un test de evaluación del potencial en emprendimiento social. Revesco, 128(1), 192-211. https://doi. org/10.5209/REVE.60207

Przepiorka, A. (2017). Psychological determinants of entrepreneurial success and life-satisfaction. Current Psychology, 36, 304-315. https:// link.springer.com/article/10.1007/ s12144-016-9419-1

Pucker, A., Dougherty, B., Jordan, L., Kwan, J., Kunnen, C. \& Srinivasan, S. (2018). Psychometric analysis of the SPEED questionnaire and CLDEQ-8. Investigative ophthalmology \& visual science, 59(8), 3307-3313. https:// doi.org/10.1167/iovs.18-24016

Razmus, W. \& Laguna, M. (2018). Dimensions of entrepreneurial success: A multilevel study on stakeholders of micro-enterprises. Frontiers in Psychology, 9, 791.

Rengifo, M. (2015). La globalización de la sociedad del conocimiento y la transformación universitaria. Revista
Latinoamericana de Ciencias, Niñez y Juventud, 13(2), 809-822.

Roca, J. (2020). Los costes de las renovables se desploman en 2020: la energía solar y la eólica terrestre son las fuentes más baratas en casi todo el mundo. El periódico de la energía. https://bit.ly/3taXkSv

Rojas, G., Pertuz, V., Navarro, A. y Quintero, L. (2019). Instrumento para identificar características personales y didáctica utilizadas por los docentes en la formación de emprendedores. Formación Universitaria, 12(2), 2940.

Rosales, R., Rivera, S., García, M. (2017). Revisión psicométrica de tres escalas del perdón: midiendo la metamorfosis emocional. Acta de investigación psicológica, 7(1), 25772584.

Sánchez, J. (2010). Evaluación de la personalidad emprendedora: validez factorial del cuestionario de orientación emprendedora (COE). Revista Latinoamericana de Psicología, 42(1), 41-52.

Sánchez, J., Ward, A., Hernández, B. y Florez, J. (2017). Educación emprendedora: Estado del arte. Propósitos y Representaciones, 5(2), 401-473. $\quad$ http://dx.doi. org/10.20511/pyr2017.v5n2.190

Shu, W., Hsiu, Y. \& Pei, W. (2019). How the new type of entrepreneurship education complements the traditional one in developing entrepreneurial competencies and intention. Frontiers in Psychology, 10, 2048. https://doi.org/10.3389/ fpsyg.2019.02048

Suárez, J. y Pedrosa, I. (2016). Evaluación de la personalidad emprendedora: situación actual y líneas de futuro. Papeles del Psicólogo, 37(1), 62-68. 
Castillo Saavedra, Ericson Felix; Reyes Alfaro, Cecilia Elizabeth;

Ayala Jara, Carmen Isolina; Arroyo Rosales, Elizabeth Madeleine

Escala de perfil emprendedor en estudiantes universitarios peruanos

Voda, A. \& Florea, N. (2019). Impact of personality traits and entrepreneurship education on entrepreneurial intentions of business and engineering students. Sustainability, 11(4), 1192.

Tarapuez, E., García, M. y Castellano, N. (2015). Elementos para la construcción de un instrumento que permita medir la intención empresarial en estudiantes universitarios de último semestre. Tendencias, 16(2), 209-227.

Ward, A., Hernández, B. \& Sánchez, J. (2019). Entrepreneurial potential and gender effects: the role of personality traits in university students' entrepreneurial intentions. Frontiers

in Psychology, 10, 1-18. https://doi. org/10.3389/fpsyg. 2019.02700

Zapata, A., Corredor, A. y Mena, A. (2019). Emprendimiento y cultura en instituciones universitarias: Una revisión de la literature. Revista Venezolana de Gerencia, 24(85), 8392. https://produccioncientificaluz. org/index.php/rvg/article/ view/23830/24261

Zapata, G. y Hernández, A. (2017). Escala de medición de la percepción gerencial y directiva del entorno empresarial. Revista de Ciencias Sociales (Ve), 23(2), 84-99. https:// doi.org/10.31876/rcs.v23i2.24954 